\title{
Evidence theory and differential evolution based uncertainty quantification for buckling load of semi-rigid jointed frames
}

\author{
HESHENG TANG ${ }^{1,2, *}$, YU SU ${ }^{2}$ and JIAO WANG ${ }^{2}$ \\ ${ }^{1}$ State Key Laboratory for Disaster Reduction in Civil Engineering, \\ Tongji University, Shanghai 200092, China \\ ${ }^{2}$ Research Institute of Structural Engineering and Disaster Reduction, \\ College of Civil Engineering, Tongji University, Shanghai 200092, China \\ e-mail: thstj@tongji.edu.cn
}

MS received 18 August 2014; revised 30 March 2015; accepted 2 April 2015

\begin{abstract}
The paper describes a procedure for the uncertainty quantification (UQ) using evidence theory in buckling analysis of semi-rigid jointed frame structures under mixed epistemic-aleatory uncertainty. The design uncertainties (geometrical, material, strength, and manufacturing) are often prevalent in engineering applications. Due to lack of knowledge or incomplete, inaccurate, unclear information in the modeling, simulation, measurement, and design, there are limitations in using only one framework (probability theory) to quantify uncertainty in a system because of the impreciseness of data or knowledge. Evidence theory provides an alternative to probability theory for the representation of epistemic uncertainty that derives from a lack of knowledge with respect to the appropriate values to use for various inputs to the model. Unfortunately, propagation of an evidence theory representation for uncertainty through a model is more computationally demanding than propagation of a probabilistic representation for uncertainty. In order to alleviate the computational difficulties in the evidence theory based UQ analysis, a differential evolution-based computational strategy for propagation of epistemic uncertainty in a system with evidence theory is presented here. A UQ analysis for the buckling load of steelplane frames with semi-rigid connections is given herein to demonstrate accuracy and efficiency of the proposed method.
\end{abstract}

Keywords. Evidence theory; uncertainty quantification; buckling load; semi-rigid jointed frame structures; differential evolution.

\section{Introduction}

In steel-frame structures, stability is very important because when a structure loses its stability, the failure is abrupt, there is no way to predict it, and the damage is always serious. Buckling

*For correspondence 
is a critical issue for structural stability in structural design. However, design uncertainties (applied loads, geometrical, material, strength, and manufacturing) are often prevalent in practical engineering structures. As a consequence, the buckling load may also be strongly affected by the uncertainties of the structural parameters. The sensitivity of the buckling load to structural parameters has long been investigated in structural design, determining that uncertain considerations are unavoidable when stability problems are of concern. As reported, the buckling load or the equivalent buckling length factor of steel-frame structures is greatly influenced by stiffness of bracing elements and semi-rigid connections (Goto \& Miyashita 1998; Raftoyiannis 2005; Lien et al 2012). Moreover, many studies (Tucker \& Bennett 1990; Rauscher \& Gerstle 1992; Gao \& Haldar 1995; Sakurai \& Ellingwood 2001; Huh \& Haldar 2001) indicated that the stiffness of semi-rigid connections is actually uncertain, and this can influence the behavior of the structure significantly. Therefore, the uncertainty of the rigidity of the connections has to be taken into account in the analysis and design. In this paper, we consider the uncertainty of the initial rotation stiffness of supports, beam-to-column connections, and horizontal bracing.

Theoretically, the initial rotational stiffness of beam-column semi-rigid connections is determined by five main parts: tensile rigidity of the column web, compression rigidity of the column web, shear rigidity of the column web, flexural rigidity of the column flange, and flexural rigidity of the end plate. These parameters are related to the modulus of material and dimensions of joints, bolts, and welds (Moncarz \& Gerstle 1981; Yee \& Melchers 1987; Azizinamini et al 1987; Eurocode 3 2005). In reality, some of these design parameters are disregarded, which can lead to uncertainties of the stiffness of the connections. In most analyses of buckling, structural properties and applied loads are considered certain, which ignores the fact that imperfections and unknown changes in properties, albeit small, are required for the onset of buckling.

Different approaches are used for buckling analysis when considering uncertainties such as probability theory, fuzzy theory, convex models, and interval calculations. Hadianfard \& Razani (2003) considered the loads and the resistance of members to be random variables and adopted Monte Carlo simulation to estimate the probability of failure of a steel frame with semi-rigid connections. Modares et al (2004) used an interval finite element method to analyze buckling of structures with uncertain properties and loads. Alibrandi et al (2009) presented a response surface method for the evaluation of the probabilistic buckling analysis. Korkmaz et al (2011) employed fuzzy logic algorithm to investigate the material uncertainties on column design and proposed an uncertainty model for critical column buckling reinforced concrete buildings. Basaga et al (2012) applied a combination of the reliability method and finite element method to determine the reliability indexes of steel-braced reinforced concrete frames with semi-rigid connections.

To represent the uncertainty in a system, Helton \& Oberkampf (2004) and Oberkampf \& Helton (2001) described uncertainties as two distinct types: aleatory uncertainty and epistemic uncertainty. Aleatory uncertainty is also called irreducible and inherent uncertainty. Epistemic uncertainty is subjective and reducible uncertainty that stems from lack of knowledge or data. In engineering, the problem parameters (geometrical, material, strength, and manufacturing) are given or considered with uncertainties. In general, the sources of aleatory uncertainty are typically represented using a probabilistic framework under which the aleatory uncertainty can be represented by a finite number of random variables with some known distribution. However, when sufficient data are not available or there is lack of information due to ignorance, the classical probability methodology may not be suitable. In comparison to the quantification of aleatory uncertainty, the analysis of epistemic uncertainty has proven more challenging. Several theories to handle epistemic uncertainty have been proposed in the literature including possibility theory (Dubois \& Prade 1996; 
Byeng et al 2007; Du et al 2006; Hanss \& Turrin 2010), interval theory (Muhanna \& Mullen 2001; Ben-Haim 2006; Li \& Azarm 2008), evidence theory (Shafer 1976; Bae \& Ramana 2004; Limbourg \& Rocquigny 2010; Helton et al 2010; Jiang et al 2013 etc. Among these methods, evidence theory has the greatest potential in uncertainty quantification since it is more general than probability and possibility theories. It uses plausibility and belief to measure the likelihood of an event, without making additional assumptions. Contrary to the classical probability theory, possibility theory is usually only used to quantify epistemic uncertainty.

Evidence theory has a strong ability to deal with uncertainty modeling and decision under uncertainty, when the evidence about uncertainty is imprecise. However, the large computational cost caused by its discrete property severely influences the practicability of evidence theory. There is no clear function expression for unclear information in evidence theory, which is different from probability density function and possibility distribution function (membership function of fuzzy variable). As the uncertainty variable is represented by many discontinuous sets instead of smooth and continuous explicit function, onerous computational cost is inevitable in uncertainty quantification with evidence theory.

Uncertainty propagation based on evidence theory is a very important step in dealing with uncertainty, which is to find the maximum and minimum values of the system response, and this part can be very computationally expensive. There are two main approaches to solving this problem: sampling (Walpole 1998) and optimization (Storn \& Price 1997; Tang et al 2008). In the sampling method, computational complexity will increase exponentially with the increasing of uncertainty variable and quantity of the uncertainty interval; moreover, result accuracy is highly dependent on the number of samples and the number of interval combinations. In order to alleviate the computational difficulties in the evidence theory based uncertainty quantification (UQ) analysis, a differential evolution-based computational strategy for the propagation of epistemic uncertainty in a system with evidence theory is developed. Moreover, based on the flexibility of evidence theory, evidence theory is used to integrate aleatory and epistemic uncertainty of parameters into a unified framework for representation and propagation in this paper. The buckling load or the equivalent buckling length factor of the steel-frame structures is greatly influenced by the stiffness of the bracing elements and semi-rigid connections. The design uncertainties (initial connections' rigidity, bracing rigidity, and applied loads) are taken into consideration. In this paper, we are concerned with the epistemic uncertainty where sparse and/or imprecise information is available regarding a stochastic quantity; as a result, the distribution type is assumed to be known, but the distribution parameters are uncertain. An uncertainty quantification analysis for the buckling load of steel-plane frames with semi-rigid connections is presented herein to demonstrate the accuracy and efficiency of the proposed method.

\section{Buckling analysis of steel frames with semi-rigid connections}

The present work deals with the uncertainty quantification on the buckling load of an $n$-span steel-plane frame with semi-rigid connections, supports, and elastic bracing as shown in figure 1 . The method of the simplified joint modeling prescribed by Raftoyiannis (2005) and Eurocode 3 (2005) is adopted herein and a linear moment-rotation relation accounting for joint flexibility is incorporated into the buckling analysis of the frames. In figure 1, the beam-column connections are extended end-plate connections. Each member is assumed to be an element. The moment of inertia of each member is $I_{i}(i=1 \sim 2 n+1), P_{i}(i=1 \sim n+1)$ is the concentrated load on each top of the column, and $S_{i}(i=1 \sim n+1)$ is the secant stiffness of the support connection. $S_{L i}, S_{R i}(i=1 \sim n)$ is the secant stiffness of the left and right beam-to-column connection. A 


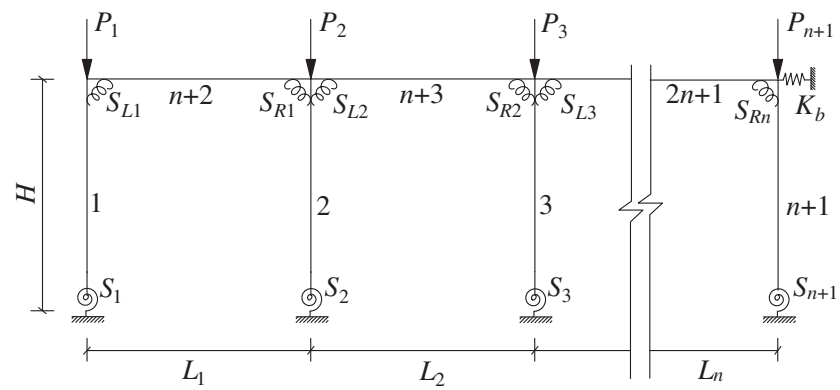

Figure 1. Analytical model of the $n$-span steel frame with semi-rigid connections.

horizontal spring with axial stiffness $K_{b}$ is used, accounting for lateral elastic support such as bracings. A linear buckling analysis can be performed, because on the onset of buckling the joints undergo small rotation values. Thus, the simplified moment-rotation relation can be written as follows:

$$
M_{j}=S_{j} \theta_{j},
$$

where $M_{j}=$ the moment of joint $j$ and $\theta_{j}=$ relative rotation of the connections.

Using Eq. (1), the connection flexibility can be directly introduced into the boundary conditions of the frame and a linear buckling analysis can be readily performed.

The governing differential equations for the columns and the beams are given as follows:

$$
\begin{aligned}
& v_{i}^{\prime \prime \prime \prime}+k_{i}^{2} v_{i}^{\prime \prime}=0,(i=1 \sim n+1), \\
& w_{j}^{\prime \prime \prime \prime}=0,(j=n+2 \sim 2 n+1),
\end{aligned}
$$

where $v_{i}=$ the horizontal displacement of the $i$ th column, $w_{j}=$ the vertical deflection of the $j$ th beam, and

$$
k_{i}^{2}=\frac{P_{i}}{E I_{i}},(i=1 \sim n+1) .
$$

The general solutions of Eqs. (2) and (3) are

$$
\begin{gathered}
v_{i}\left(x_{i}\right)=A_{i} \sin k_{i} x_{i}+B_{i} \cos k_{i} x_{i}+C_{i} x_{i}+D_{i}, \quad(i=1 \sim n+1), \\
w_{j}\left(x_{j}\right)=A_{j} x_{j}^{3}+B_{j} x_{j}^{2}+C_{j} x_{j}+D_{j}, \quad(j=n+2 \sim 2 n+1) .
\end{gathered}
$$

The relationship between the moment $M_{i}$, shear force $V_{i}$, and the relative horizontal displacement of any section of the column and beam can be expressed as follows:

$$
\begin{gathered}
M_{i}\left(x_{i}\right)=-E I_{i} v_{i}^{\prime \prime}\left(x_{i}\right), \quad(i=1 \sim n+1), \\
V_{i}\left(x_{i}\right)=-E I_{i} v_{i}^{\prime \prime \prime}\left(x_{i}\right)-P_{i} v_{i}^{\prime}\left(x_{i}\right), \quad(i=1 \sim n+1), \\
M_{j}\left(x_{j}\right)=-E I_{j} w_{j}^{\prime \prime}\left(x_{j}\right), \quad(j=n+2 \sim 2 n+1), \\
V_{j}\left(x_{j}\right)=-E I_{j} w_{j}^{\prime \prime \prime}\left(x_{j}\right), \quad(j=n+2 \sim 2 n+1) .
\end{gathered}
$$

Employing the frame shown in figure 1, the boundary conditions at the supports are

$$
\left\{\begin{array}{c}
v_{i}(0)=0 \\
M_{i}(0)=-E I_{i} v_{i}^{\prime \prime}(0)=-S_{i} v_{i}^{\prime}(0)
\end{array}(i=1 \sim n+1)\right.
$$


Neglecting the effect of axial deflection, the boundary conditions of the beams and columns are

$$
\begin{aligned}
& \left\{\begin{array}{c}
w_{j+n+1}(0)=0 \\
w_{j+n+1}\left(L_{j}\right)=0
\end{array}(j=1 \sim n),\right. \\
& v_{i}(H)=v_{1}(H),(i=2 \sim n+1) .
\end{aligned}
$$

Employing the boundary conditions and the kinematic conditions:

$$
\begin{gathered}
\sum_{i=1}^{n+1} V_{i}(H)+K_{b} v_{n+1}(H)=0, \\
M_{i}(H)+M_{n+i}\left(L_{i-1}\right)-M_{n+i+1}(0)=0,(i=1 \sim n+1), \\
M_{n+1}\left(L_{0}\right)=0, M_{2 n+2}(0)=0, \\
\left\{\begin{array}{c}
M_{n+1+i}(0)=S_{L i}\left[v_{i}^{\prime}(H)-w_{n+1+i}^{\prime}(0)\right] \\
M_{n+1+i}\left(L_{i}\right)=S_{R i}\left[w_{n+1+i}^{\prime}\left(L_{i}\right)-v_{i+1}^{\prime}(H)\right]
\end{array}(i=1 \sim n) .\right.
\end{gathered}
$$

We obtain the following system of non-dimensional equations:

$$
\overline{S_{i}} \alpha_{i} \beta \overline{A_{i}}+\alpha_{i}^{2} \beta^{2} \overline{B_{i}}+\overline{S_{i} C_{i}}=0,(i=1 \sim n+1),
$$

$\sin \alpha_{i} \beta \overline{A_{i}}+\left(\cos \alpha_{i} \beta-1\right) \overline{B_{i}}+\overline{C_{i}}-\sin \alpha_{i+1} \beta \bar{A}_{i+1}-\left(\cos \alpha_{i+1} \beta-1\right) \bar{B}_{i+1}-\bar{C}_{i+1}=0,(i=1 \sim n)$,

$\bar{k}_{b} \sin \alpha_{n+1} \beta \bar{A}_{n+1}+\bar{k}_{b}\left(\cos \alpha_{n+1} \beta-1\right) \bar{B}_{n+1}-\sum_{i=1}^{n} \xi_{i}^{2} \alpha_{i}^{2} \beta^{2} \bar{C}_{i}+\left(\bar{k}_{b}-\xi_{n+1}^{2} \alpha_{n+1}^{2} \beta^{2}\right) \bar{C}_{n+1}=0$,

$$
\begin{aligned}
& \xi_{i}^{2} \alpha_{i}^{2} \beta^{2} \sin \alpha_{i} \beta \bar{A}_{i}+\xi_{i}^{2} \alpha_{i}^{2} \beta^{2} \cos \alpha_{i} \beta \bar{B}_{i}-6 \bar{l}_{i-1} \eta_{i-1}^{2} \bar{A}_{n+i}-2 \eta_{i-1}^{2} \bar{B}_{n+i} \\
& \quad+2 \eta_{i}^{2} \bar{B}_{n+i+1}=0,(i=1 \sim n+1) .
\end{aligned}
$$

Notice that $\xi_{1}=1, \eta_{0}=0, \eta_{n+1}=0$.

$$
\begin{aligned}
& \bar{S}_{L i} \alpha_{i} \beta \cos \alpha_{i} \beta \bar{A}_{i}-\bar{S}_{L i} \alpha_{i} \beta \bar{A}_{i} \sin \alpha_{i} \beta \bar{B}_{i}+\bar{S}_{L i} \bar{C}_{i}+\bar{S}_{L i} \bar{l}_{i}^{2} \bar{A}_{n+i+1} \\
& \quad+\left(2+\bar{S}_{L i} \bar{l}_{i}\right) \bar{B}_{n+i+1}=0,(i=1 \sim n) \\
& \bar{S}_{R i} \alpha_{i+1} \beta \cos \alpha_{i+1} \beta \bar{A}_{i+1}-\bar{S}_{R i} \alpha_{i+1} \beta \sin \alpha_{i+1} \beta \bar{B}_{i+1}+\bar{S}_{R i} \bar{C}_{i+1} \\
& \quad-2 \bar{l}_{i}\left(3+\bar{S}_{R i} \bar{l}_{i}\right) \bar{A}_{n+i+1}-\left(2+\bar{S}_{R i} \bar{l}_{i}\right) \bar{B}_{n+i+1}=0,(i=1 \sim n)
\end{aligned}
$$

with respect to the non-dimensional constants:

$$
\begin{gathered}
\bar{A}_{i}=A_{i} / H, \bar{B}_{i}=B_{i} / H, \bar{C}_{i}=C_{i}, \bar{D}_{i}=D_{i} / H \text { and } \\
\alpha_{i}^{2}=\left(P_{i} I_{1}\right) /\left(P_{1} I_{i}\right), \beta^{2}=\left(P_{1} H^{2}\right) /\left(E I_{1}\right), \bar{S}_{i}=\left(S_{i} H\right) /\left(E I_{i}\right), \\
\bar{S}_{L i}=\left(S_{L i} H\right) /\left(E J_{i}\right), \bar{S}_{R i}=\left(S_{R i} H\right) /\left(E J_{i}\right), \bar{l}_{i}=L_{i} / H, \\
\bar{k}_{b}=\left(K_{b} H^{3}\right) /\left(E I_{1}\right), \xi_{i}^{2}=\left(E I_{i}\right) /\left(E I_{1}\right)=I_{i} / I_{1},(i=1 \sim n+1), \\
\eta_{i}^{2}=E J_{i} / E I_{1}=J_{i} / I_{1},(i=1 \sim n) .
\end{gathered}
$$

where $K_{b}=$ rigidity of the horizontal brace, which can be calculated by the parameters of bracing:

$$
K_{b}=\sum\left(E A_{d i} / L_{i}\right) \cos ^{2} \theta_{i}
$$


Apparently, there are $3(n+1)+2 n$ equations above with the number of $3(n+1)+2 n$ variables: $\bar{A}_{i}(i=1 \sim n+1), \bar{B}_{i}(i=1 \sim n+1), \bar{C}_{i}(i=1 \sim n+1), \bar{A}_{i+n+1}(i=1 \sim n), \bar{B}_{i+n+1}(i=1 \sim$ $n)$. Then, the buckling equation of the semi-rigid connections, supports, and elastic bracing frame (figure 1) with semi-rigid joints results by setting the determinant of the unknown constants of Eqs. (18)-(25) equal to zero, i.e.,

$$
\operatorname{det}\left[\mathbf{K}_{1}\right]=0 .
$$

The non-dimensional critical load $\beta_{\mathrm{cr}}$ is obtained by solving Eq. (26), and subsequently, the buckling load of the frame is computed from

$$
P_{c r}=\left(\beta_{c r}^{2} E I_{1}\right) / H^{2}=\pi^{2} E I_{1} /(K H)^{2} .
$$

From Eq. (27) results the equivalent buckling length factor $K$ of the column, which is

$$
K=\pi / \beta_{\mathrm{cr}}
$$

Equation (26) has $3(n+1)+2 n$ characteristic values, but the minimum is the one we care about here. According to the analysis model we use in this paper, the proportional relation between the forces imposed on the top of each column is

$$
P_{i}=\lambda_{i}^{2} P_{1}, i=1 \sim n+1 .
$$

We solve Eq. (26) to get the minimum characteristic value $\beta_{\min }$, then we can get the buckling load upon each column:

$$
P_{i}=\beta_{c r}^{2} E I_{i} \alpha_{i}^{2} / H^{2}, i=1 \sim n+1,
$$

where $\alpha_{i}=\lambda_{i} / \xi_{i}$.

From the analysis above, it is easy to find that $\beta_{c r}$ is mainly connected with four parameters: $S_{i}$ (the rigidity of support), $K_{b}$ (the rigidity of the horizontal rigidity), $R_{i}$ (the rigidity of beamcolumn connections), and the $\lambda_{i}$ (load factor).

\section{Evidence theory for uncertainty quantification}

\subsection{Fundamentals of evidence theory}

Evidence theory was first proposed by Dempster (1967) and extended by Shafer (1976) and is also called the Dempster-Shafer theory (DST). Among the numerous inexact probability methods, evidence theory is the most closely related to probability theory, which is a generalization of classical probability theory. It needs less information to describe the phenomenon than probability theory and it uses the D-S combination rule instead of Bayes formula to update the belief function.

Four important concepts are defined in evidence theory: frame of discernment (FD), basic belief assignment (BBA), belief function (Bel), and plausibility function (Pl). The frame of discernment consists of all finite elementary propositions for an uncertain variable and is denoted by $\Theta$. The power set of $\Theta\left(2^{\Theta}\right)$ indicates all the possible subset propositions of $\Theta$. As a primitive definition in DST, the basic belief assignment is assigned through a mapping function $\mathrm{m}: 2^{\Theta} \rightarrow$ $[0,1]$ to express the degree of belief in a proposition. This mass function is given as

$$
\left\{\begin{array}{c}
m(\phi)=0 \\
\sum_{\mathrm{A} \subseteq \Theta} m(\mathrm{~A})=1 .
\end{array}\right.
$$




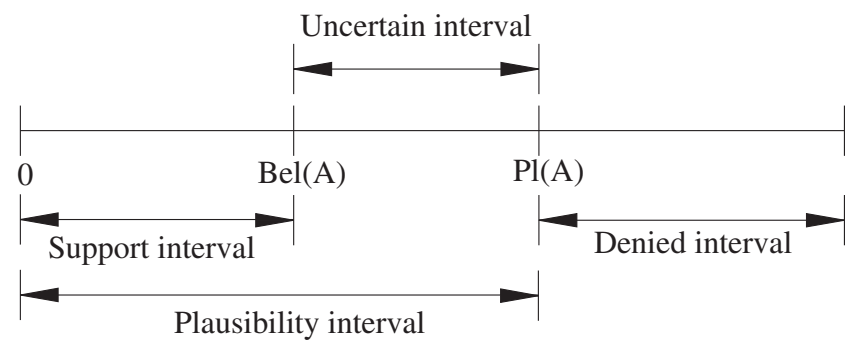

Figure 2. Uncertainty description of proposition A.

Every subset $\mathrm{A} \subseteq \Theta$ for which $m(\mathrm{~A})>0$ is called a focal element. In evidence theory, each focal element is usually expressed by multiple intervals - scattered, nested, or partially overlapped interval-which is consistent with the given insufficient and imprecise information. Given the basic belief assignment (BBA) $m$, the belief measure and plausibility measure are uniquely determined by

$$
\begin{aligned}
& \operatorname{Bel}(\mathrm{A})=\sum_{\mathrm{B} \subseteq \mathrm{A}} m(\mathrm{~B}) \\
& \mathrm{Pl}(\mathrm{A})=1-\operatorname{Bel}(\overline{\mathrm{A}})=\sum_{\mathrm{B} \cap \mathrm{A} \neq \phi} m(\mathrm{~B}) .
\end{aligned}
$$

The belief Bel(A) is interpreted to be the total evidence corresponding to all the subsets of A, while the plausibility $\mathrm{Pl}(\mathrm{A})$ represents the sum of $\mathrm{BBA}$ values corresponding to all the sets $\mathrm{B}$ intersecting with A. Apparently, Bel(A) means the confident degree to believe that event A is true, and $\mathrm{Pl}(\mathrm{A})$ means the confident degree to believe that event $\mathrm{A}$ is not false, thus $\mathrm{Pl}(\mathrm{A})$ $\geq \operatorname{Bel}(\mathrm{A})$. For information from multiple sources, the combined evidence can be obtained by Dempster's rule of combination (Shafer 1976). Readers are referred to comprehensive literature on the subject (e.g., Sentz \& Ferson 2002) for a detailed discussion.

$\mathrm{Bel}(\mathrm{A})$ and $\mathrm{Pl}(\mathrm{A})$ also act as upper and lower bounds of classical probability to measure the likelihood of events without use of the explicit probability distribution, defining an intervalvalued probability distribution, $[\operatorname{Bel}(\mathrm{A}), \mathrm{Pl}(\mathrm{A})]$, not a single probability distribution, as shown in figure 2. It is also called the uncertain interval for event $\mathrm{A} . \mathrm{Pl}(\mathrm{A})-\mathrm{Bel}(\mathrm{A})$ indicates that we are uncertain about whether event $\mathrm{A}$ is true or not. The existence of epistemic uncertainty is due to lack of knowledge or information, so the confident degree of event A cannot represent

the confident degree of $\tilde{A}$, i.e., $m(A)+m(\tilde{A}) \leq 1$, which is completely different from the single probability distribution in probability theory, i.e., $\mathrm{p}(\mathrm{A})+\mathrm{p}(\tilde{\mathrm{A}})=1$. Therefore, traditional probability theory is a special case of evidence theory.

\subsection{Evidence theory for uncertainty quantification using differential evolution}

The previous section described uncertainty qualification based on evidence theory. This section shows how to propagate uncertainty through a given model (transfer function). This will be shown using figure 3 (here only one uncertain variable is considered).

In this figure, $x$ is uncertain variable, $d$ is certain variable, and $f$ is a system model (system response). Essentially, uncertainty propagation is used to determine the uncertainty of system response when the system uncertain input is given. 


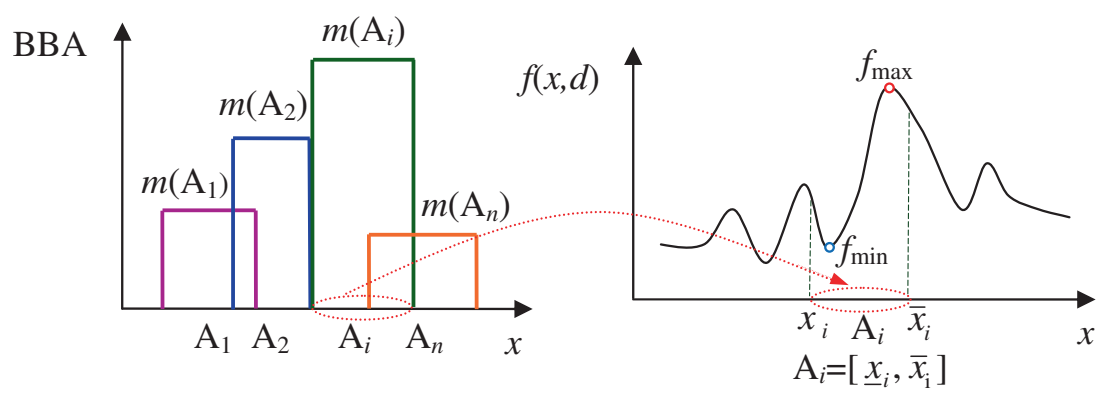

Figure 3. Propagation of system uncertainty.

As mentioned previously, the uncertainty variable is usually expressed as a series of intervals based on limited information, where $n$ variables will form an $n$-dimensional hypercube. Therefore, uncertainty propagation is a process of finding the maximum and minimum of the response value in each hypercube, as figure 3 illustrates, and we will solve two related bound-constrained problems:

$$
\left[f_{\min }, f_{\max }\right]=\left[\min f\left(x_{i}\right), \max f\left(x_{i}\right)\right] \text { subject to } \underline{x}_{i} \leq x_{i} \leq \bar{x}_{i} .
$$

Through the above analysis it can be found that the computational cost of the uncertainty propagation analysis using evidence theory is mainly determined by two factors: the number of the focal elements and the search of extreme values of the system response over each focal element. It can be very computationally expensive due to the fact that the quantity of the focal element is large and system function form is complex in practical engineering, so the conventional optimization algorithm cannot solve this problem. To alleviate this computational burden, a modern intelligent optimization method based on differential evolution (DE) is proposed here. The DE method is a good choice for such a task since the uncertainty quantification (UQ) process requires an optimization algorithm that is derivative-free and can handle discrete plausibility values.

Differential evolution is a stochastic population-based search method proposed by Storn \& Price (1997) for solving non-linear, high-dimensional, and complex computational optimization problems. As a novel evolutionary computation technique, differential evolution resembles the structure of an evolutionary algorithm (EA), but differs from traditional EAs in its generation of new candidate solutions and by its use of a "greed selection scheme. The characteristics together with other mechanisms of DE make it a fast and robust algorithm and an alternative to EA. The basic concepts and notations of differential evolution are briefly introduced in the following.

An optimization task consisting of $n$ parameters can be represented by an $n$-dimensional vector. Let $S \in R^{\mathrm{n}}$ be the search space of the problem. Then, the $n$-dimensional vector can be represented by $\boldsymbol{x}_{i}=\left(x_{i 1}, x_{\mathrm{i} 2}, \ldots, x_{i n}\right)^{\mathrm{T}} \in S, i=1,2,3, \ldots$, NP. In DE, a population of NP (population size) candidate solution vectors is initialized randomly at the start, which is evolved to find optimal solutions through the mutation, crossover, and selecting operation procedures.

Mutation is used to enable search diversity in the parameter space as well as to direct the existing object vectors with a suitable amount of parameter variation in a way that will lead to better results at a suitable time. It keeps the search robust and explores new areas in the search domain.

According to the mutation operator, for each individual, $x_{i}^{G}, i=1,2, \ldots, \mathrm{NP}$, at generation $G$, a mutation vector $v_{i}^{(G+1)}=\left(v_{i 1}^{(G+1)}, v_{i 2}^{(G+1)}, \ldots, v_{i n}^{(G+1)}\right)$ is determined using different 
mutation methods. The mutation method of DE/current-to-best/1/bin recommended by Storn \& Price (1997) is used in this paper to deal with the uncertainty propagation in evidence theory.

$$
v_{i}^{(G+1)}=x_{1}^{(G)}+F_{1}\left(x_{\text {best }}^{(G)}-x_{i}^{(G)}\right)+F\left(x_{r 1}^{(G)}-x_{r 2}^{(G)}\right),
$$

where $\boldsymbol{x}_{\text {best }}^{G}$ is the best individual of the population at generation $G ; F$ and $F_{1}>0$, called mutation constants, control the amplification of difference between two individuals so as to avoid search stagnation; and $r_{1}, r_{2}$ are mutually different integers, randomly selected from set $\{1,2, \ldots, i-1$, $i+1, \ldots, \mathrm{NP}\}$.

Following the mutation phase, the crossover operator is applied on the population. For each mutant vector, $v_{i}^{(G+1)}$, the trial vector $\boldsymbol{u}_{i}^{(G+1)}=\left(u_{i 1}^{(G+1)}, u_{i 2}^{(G+1)}, \ldots, u_{i n}^{(G+1)}\right)^{\mathrm{T}}$ is generated, with

$$
u_{i j}^{(G+1)}=\left\{\begin{array}{c}
v_{i j}^{(G+1)} \text { if }(r(j) \leq C R) \text { or }(j=r n(i)) \\
x_{i j}^{(G)} \text { if }(r(j)>C R) \text { or }(j \neq r n(i))
\end{array},\right.
$$

where $j=1,2, \ldots, n ; r(j)=\operatorname{rand}(j)$ and $r n(i)=\operatorname{rand} n(i) ; \operatorname{rand}(j)=j$ th independent random number uniformly distributed in the range of $[0,1]$. randn $(i)$ is a randomly chosen index from the set $\{1,2, \ldots, n\}$, and $C R$ is a user-defined crossover constant $\in[0,1]$ that controls the diversity of the population.

DE employs a greedy criterion that is different from genetic algorithms. After producing the offspring, the performance of the offspring vector and its parent is compared and the better one is selected. The selection process is represented by the following equation:

$$
\boldsymbol{x}_{i}^{(G+1)}=\left\{\begin{array}{l}
\boldsymbol{u}_{i}^{(G+1)} \text { if }\left(f\left(\boldsymbol{u}_{i}^{(G+1)}\right)<f\left(\boldsymbol{x}_{i}^{(G)}\right)\right) \\
\boldsymbol{x}_{i}^{(G)} \text { otherwise }
\end{array} .\right.
$$

Thus, each individual of the trial vector is compared with its parent vector and the better one is passed to the next generation, so the best individuals in the population are preserved. These steps are repeated until specified stop criterion is reached. Meanwhile, the optimal results can be obtained. In this section, the DE approach is briefly described. A detailed survey of the DE family of algorithms can be found in Das \& Suganthan (2011).

\subsection{Procedures of evidence-based UQ for buckling analysis using DE}

The evidence theory is a well-suited framework for representing both epistemic and aleatory uncertainty. Evidence theory used to integrate aleatory and epistemic uncertainty of parameters into a unified framework for representation and propagation will be addressed in this section.

The procedures of uncertainty quantification for buckling analysis of steel frames with semirigid connections using evidence theory and differential evolution-based computational strategy can be summarized as follows:

Step 1: Getting parameter information and identifying uncertainty type.

Two types of uncertainty (aleatory and epistemic uncertainty) of the three main variables, $R$, $u_{k}$, and $\lambda$ are included in this study. For the aleatory parameter, the equivalent BBA values within the specified intervals are equal to the areas under the probability density distribution function. 
Step 2: Constructing the BBA structures.

After obtaining the BBA structures of all the uncertain parameters, we can integrate them into a joint structure, which is defined by the Cartesian product of the combined BBA structures for each uncertain parameter.

$$
\left.C=R \times \mu_{k} \times \lambda=\left\{c_{i j k}=R_{i} \times \mu_{k j} \times \lambda_{k}\right], c_{i j k} \subset C\right\} .
$$

The result of the Cartesian product indicates all possible joint sets that construct the joint frame of discernment.

Step 3: Calculating Bel and Pl.

Every element of the Cartesian set $C$ is required to be checked in the evaluation of the belief and plausibility functions by finding the minimal and maximal responses of the system (buckling equation (26)) using DE. The belief is obtained by summing all the joint basic belief assignment of element set that agree with the event partially. The plausibility function is calculated by summing BBAs that agree with the event totally or partially.

\section{Numerical examples}

In order to demonstrate the efficiency of the proposed UQ methodology in evaluating the buckling loads $P_{\mathrm{cr}}$ of a frame when the design uncertainties are considered for a more practical engineering application of the buckling behavior of realistic steel frames, two examples of steel frame with semi-rigid connections are presented to demonstrate the validity of the forgoing proposed method.

\subsection{Two-span single storey braced steel frame}

Figure 4 shows the geometry of the two-span single storey steel frame, which is based on semi-rigid supports, connections, and bracing and has the following properties: the section of beam is HN350 $\times 175 \times 7 \times 11$ with moment of inertia $1.37 \times 10^{8} \mathrm{~mm}^{4}$. The section of column is HW $200 \times 200 \times 8 \times 12$ with moment of inertia $4.47 \times 10^{7} \mathrm{~mm}^{4}$. The brace member is L63 $\times 6$ and the section area is $A_{\mathrm{d}}=7.29 \times 10^{2} \mathrm{~mm}^{2} . L_{1}=L_{2}=5000 \mathrm{~mm}, H=4000 \mathrm{~mm}$. Without loss of generality, we assume that the applied loads are $P_{i}=\lambda_{i} P(i=1 \sim 3), \lambda_{1}=\lambda_{3}=1, \lambda_{2}=\lambda$,

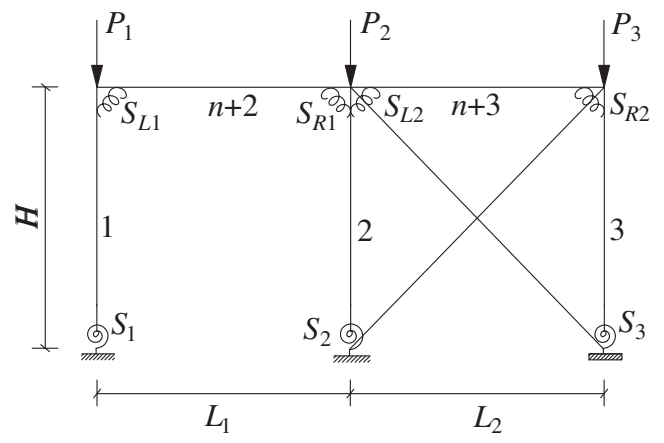

Figure 4. Two-span rectangular frames with semi-rigid supports and connections. 
Table 1. Normal distribution variables with uncertain parameters.

\begin{tabular}{lccc}
\hline Uncertain variables & Parameters & Focal elements & BBA \\
\hline$R$ & $\mu$ & {$[5,8][7,10][9,12] \times 10^{10}(\mathrm{~N} . \mathrm{mm} / \mathrm{rad})$} & $0.20,0.6,0.2$ \\
& $\sigma$ & {$[5,8][8,9][9,12] \times 10^{9}$} & $0.28,0.44,0.28$ \\
$u_{k}$ & $\mu$ & {$[0.5,0.7][0.7,0.8][0.8,1.0]$} & $0.22,0.56,0.22$ \\
& $\sigma$ & {$[0.01,0.05][0.05,0.07][0.07,0.11]$} & $0.20,0.60,0.20$ \\
& $\mu$ & {$[0.8,0.95][0.95,1.01][1.01,1.05][1.05,1.2]$} & $0.16,0.34,0.34,0.16$ \\
& $\sigma$ & {$[0.01,0.04][0.04,0.05][0.05,0.06][0.06,0.09]$} & $0.16,0.34,0.34,0.16$ \\
\hline
\end{tabular}

the stiffness of beam-to-column connections are $S_{L 1}=S_{L 2}=S_{R 1}=S_{R 2}=R$, the stiffness of support are $S_{1}=S_{2}=S_{3}=R$, and the stiffness of horizontal bracing are $K_{b}=u_{k} * K_{b}^{0}$, where $K_{b}^{0}=16,294 \mathrm{~N} / \mathrm{mm}, u_{k}$ represents the coefficient of the horizontal bracing rigidity.

Two types of uncertainty (aleatory and epistemic uncertainty) of the three main variables, $R, u_{k}$, and $\lambda$ are included in this numerical example. More specifically, two common cases are considered here: One may know the form of the probability distribution for all uncertain variables, but not be sure of the parameters governing the distribution, which is also called mixed uncertainty, while the other situation is that some of the uncertain variables are described by the above mixed uncertainty, and some are epistemic uncertainty.

Case study I

Uncertainty variables $R, u_{k}$, and $\lambda$ are supposed to follow normal distribution with the uncertain parameters $(\mu, \sigma)$, and the corresponding BBA values of the distribution parameters are given in table 1. However, in order to facilitate comparison with probability theory, the certain distribution parameter with $R \sim \mathrm{N}\left(8.5 \times 10^{10}, 9 \times 10^{9}\right) \mathrm{N} . \mathrm{mm} / \mathrm{rad}, u_{k} \sim \mathrm{N}(0.75,0.06), \lambda \sim \mathrm{N}(1,0.05)$ is also assumed in this example.

The cumulative belief ( $\mathrm{CBF}$ ) and plausibility (CPF) and the cumulative distribution functions $(\mathrm{CDF})$ of the buckling load based on evidence theory and probability theory are shown in figure 5. It is convenient to use probability theory when knowledge of the uncertain variables is thorough and the probability distribution is known exactly, and thus the corresponding result is

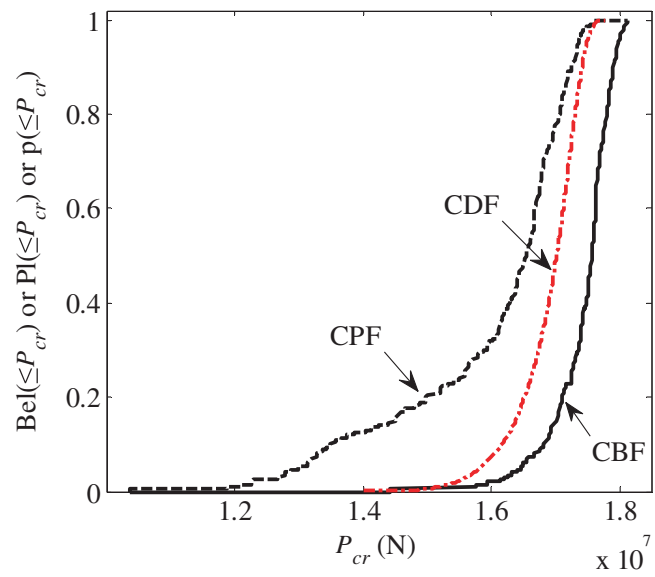

Figure 5. The buckling load cumulative distributions of a two-span steel frame based on evidence theory and probability theory in case I. 
Table 2. Comparison of results of evidence theory and probability theory in case I.

\begin{tabular}{lcc}
\hline Result information & Probability theory & Evidence theory \\
\hline Expected value of buckling load & $16,881 \mathrm{kN}$ & {$[16,001,17,424] \mathrm{kN}$} \\
Probability of buckling load, which is less than 16,000 kN & 0.07 & {$[0.02,0.32]$} \\
Buckling load, which has 95\% guarantee probability & $17,480 \mathrm{kN}$ & {$[17,347,17,951] \mathrm{kN}$} \\
\hline
\end{tabular}

also probability distribution. If the information is not enough to get the probability distribution of the uncertain variable, then each single value can be replaced by several estimated intervals with the basic belief assignment to describe the chance that the parameter falls in the interval, which is convenient to be handled by evidence theory in this case. The calculation results show that the result calculated by the probability theory is just a curve inside the region enclosed by the belief function and the plausibility function, so probability theory is just a special case of evidence theory when the focal element degenerate single value.

Table 2 shows some information of interest from the results calculated by probability theory and evidence theory. The expected values of the buckling load (mean value of the results) show that the result of evidence theory is more conservative than probability theory because of the imprecise information. The probability is $7 \%$ when the buckling load is less than $16,000 \mathrm{kN}$. The evidence theory results show that the confidence value is [0.02,0.32], it demonstrates that the probability is no more than $98 \%$ when the buckling load exceeds $16,000 \mathrm{kN}$, while the probability theory result is $93 \%$. The uncertain variables without exact probability distribution are suitable for evidence theory to handle, since it avoids the error caused by probability theory effectively; for example, the result calculated by probability theory shows that the probability is no more than $5 \%$ when the buckling load exceeds $17,480 \mathrm{kN}$, while the result in the evidence theory is $17,951 \mathrm{kN}$.

\section{Case study II}

For the second case, the $R, u_{k}$, and $\lambda$ are still considered to be uncertain, and $\lambda$ is treated as mixed uncertainty as before In addition, it is assumed that $R$ and $u_{k}$ cannot be described probabilistically due to the lack of data and knowledge, thus these two variables are epistemic, and only the multiple interval information along with the corresponding BBA values is available as given in table 3 . For comparison with probability theory, the approximate PDFs of the uncertain variables $R$ and $u_{k}$ are obtained by the assumption that the probability mass (BBA) in each interval is distributed uniformly (Savage 1972). The corresponding PDFs of $R$ and $u_{k}$ as well as the deterministic distribution of $\lambda$ are given in figure 6 .

Figure 7 shows CPF, CBF, and CDF of the buckling load based on the proposed method and probability theory with the uniform assumption, and some information of interest as in the

Table 3. BBA structure for $R, u_{k}$, and $\lambda$.

\begin{tabular}{|c|c|c|c|}
\hline $\begin{array}{l}\text { Uncertain } \\
\text { variables }\end{array}$ & & Focal elements & BBA \\
\hline$R$ & & {$[5,8][7,10][9,12] \times 10^{10} \mathrm{~N} \cdot \mathrm{mm} / \mathrm{rad}$} & $0.20,0.6,0.2$ \\
\hline$u_{k}$ & & {$[0.5,0.7][0.7,0.8][0.8,1.0]$} & $0.20,0.60,0.20$ \\
\hline \multirow{2}{*}{$\lambda$} & $\mu$ & {$[0.8,0.95][0.95,1.01][1.01,1.05][1.05,1.2]$} & $0.16,0.34,0.34,0.16$ \\
\hline & $\sigma$ & {$[0.01,0.04][0.04,0.05][0.05,0.06][0.06,0.09]$} & $0.16,0.34,0.34,0.16$ \\
\hline
\end{tabular}



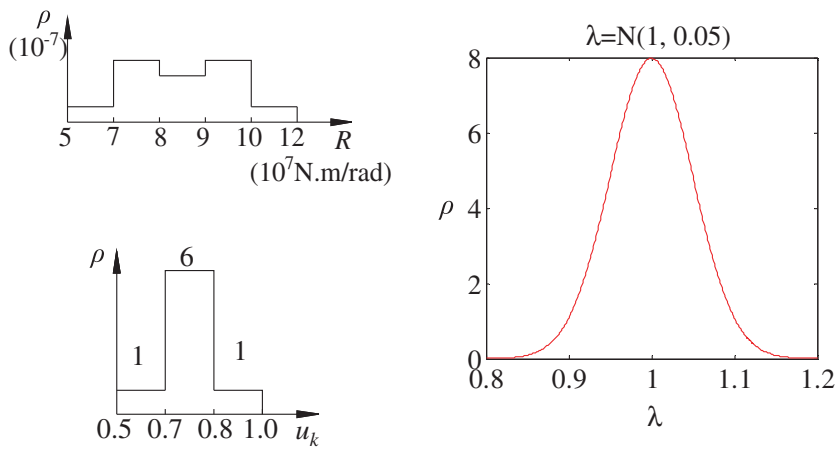

Figure 6. PDFs of $R, u_{k}$, and $\lambda$.

first case is listed in table 4. As a result, evidence theory presents the lower bound 0.03 probability and upper bound 0.50 probability of the buckling load as less than $16,000 \mathrm{kN}$, which includes the probabilistic result 0.16 . This naturally reveals that evidence theory is more intuitive and reasonable than probability theory to handle uncertainty from a lack of knowledge and information.

\subsection{Two-span two storey unbraced steel frame}

This example considers a two-span and two storey steel frame, in which the beam-to-column connections are semi-rigidly connected and the column base are rigidly connected as shown in figure 8. The geometrical property of each member is as follows: the section of column $C_{1} \sim C_{6}$ is HN500 $\times 250 \times 6 \times 8 \mathrm{~mm}$ with moment of inertia $2.99 \mathrm{e} 10^{8} \mathrm{~mm}^{4}$, the section of beam $B_{1}$ is $\mathrm{HN} 500 \times 180 \times 6 \times 8 \mathrm{~mm}$ with moment of inertia $2.31 \mathrm{e} 8 \mathrm{~mm}^{4}$, the section of beam $B_{2}$ is $\mathrm{HN} 450 \times 200 \times 6 \times 8 \mathrm{~mm}$ with moment of inertia $1.97 \mathrm{e} 8 \mathrm{~mm}^{4}$, the section of beam $B_{3}$ and $B_{4}$ is HN550 $\times 200 \times 6 \times 8 \mathrm{~mm}$ with moment of inertia $3.11 \mathrm{e} 8 \mathrm{~mm}^{4}$. In order to facilitate the calculation, the semi-rigid behavior of all the beam-column connections is assumed to be equal.

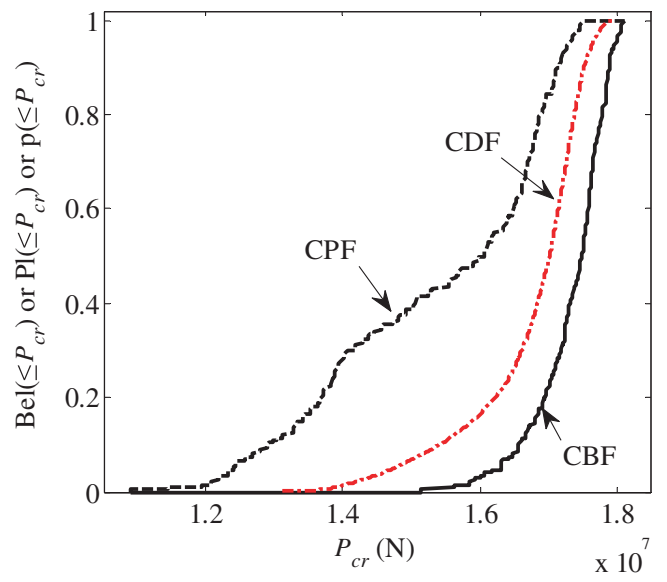

Figure 7. The buckling load cumulative distributions of a two-span steel frame based on evidence theory and probability theory in case II. 
Table 4. Comparison of results of evidence theory and probability theory in case II.

Result information

Expected value of buckling load

Probability of buckling load, which is less than $16,000 \mathrm{kN}$

Buckling load, which has $95 \%$ guarantee probability
Probability theory

$16,740 \mathrm{kN}$

0.16

$17,640 \mathrm{kN}$
Evidence theory

$[15,372,17,372] \mathrm{kN}$

$[0.03,0.50]$

$[17,282,17,971] \mathrm{kN}$

In this example, the modulus of elasticity $E$ and the rigidity of beam-column connections $R$ are considered to be epistemic uncertainty, the corresponding BBA structure is given in table 5 . For this more complex steel frame, the professional FEM software ABAQUS is selected to conduct the buckling analysis. The CBF and CPF of buckling load obtained by combining ABAQUS with evidence based UQ are shown in figure 9. The results are compared with a special case, in which the two parameters assumed to satisfy uniform distribution in each interval, and the Monte Carlo Simulation (MCS) and software ABAQUS is brought to obtain probabilistic information.

Similar conclusions as previous example are reached, the CDF curves shown in figure 9 is right between the belief and plausibility curves, for example, the median buckling load of probabilistic result in MCS calculation is $7.35 \times 10^{6} \mathrm{~N}$, and the corresponding buckling displacement of this steel frame obtained by ABAQUS is shown in figure 10, while the evidence result is a bound value of $[7.22,7.423] \times 10^{6} \mathrm{~N}$, which verifies the basic law that belief and plausibility are the lower and upper bounds of probabilistic result is accurate. Compared with the previous example, the CPF and CBF curves of the buckling load have a stair-step shape, and the "stairstep" seems more dispersed than those in figure 5 and figure 7 , which indicates more vague upper and lower probability bounds of buckling load when uncertainties are all epistemic with interval information. In addition, the results in this example suggest that the present UQ method is also valid for the complex structure by using ABAQUS to accomplish the buckling analysis during uncertainty propagation.

As discussed throughout these two examples, both fuzzy intervals and probability distribution functions can be modeled by a BBA structure in evidence theory due to its flexibility. That is, different types of information (mixed or epistemic) can be incorporated into one framework to quantify uncertainty without any unnecessary assumptions. Also, the UQ analysis for the more

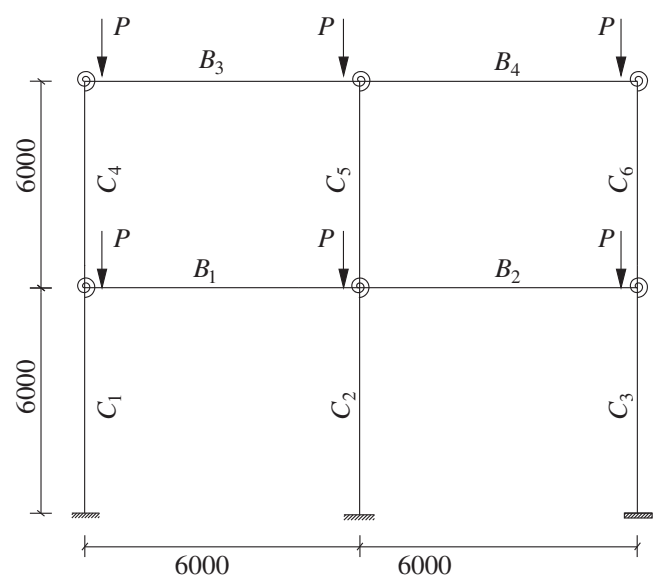

Figure 8. Two-span two storey steel frame with semi-rigid connections. 
Table 5. BBA structure for $R$ and $E$.

\begin{tabular}{lcc}
\hline Uncertain variables & Focal elements & BBA \\
\hline$R$ & {$[7,8.5][8,9.5][9,10][9.5,11] \times 10^{10} \mathrm{~N} . \mathrm{mm} / \mathrm{rad}$} & $0.1,0.5,0.3,0.1$ \\
$E$ & {$[2.02,2.05][2.05,2.06][2.06,2.07][2.07,2.1] \times 10^{5} \mathrm{MPa}$} & $0.16,0.34,0.34,0.16$
\end{tabular}

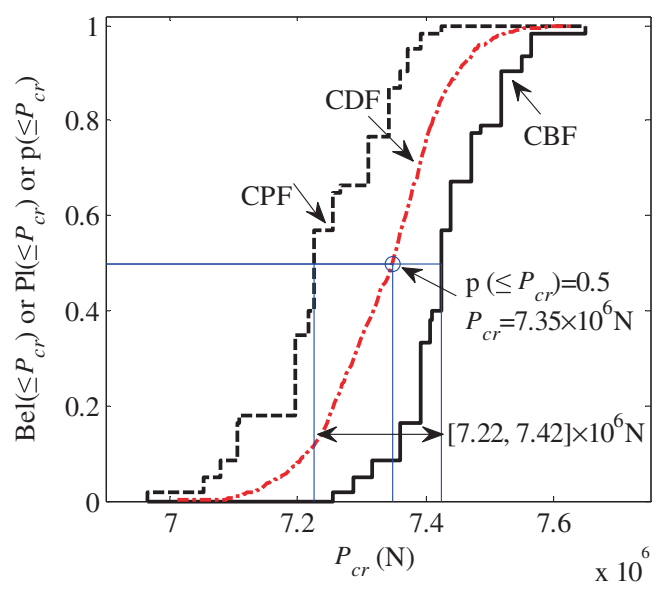

Figure 9. The buckling load cumulative distributions of a two-span two story steel frame based on evidence theory and probability theory.

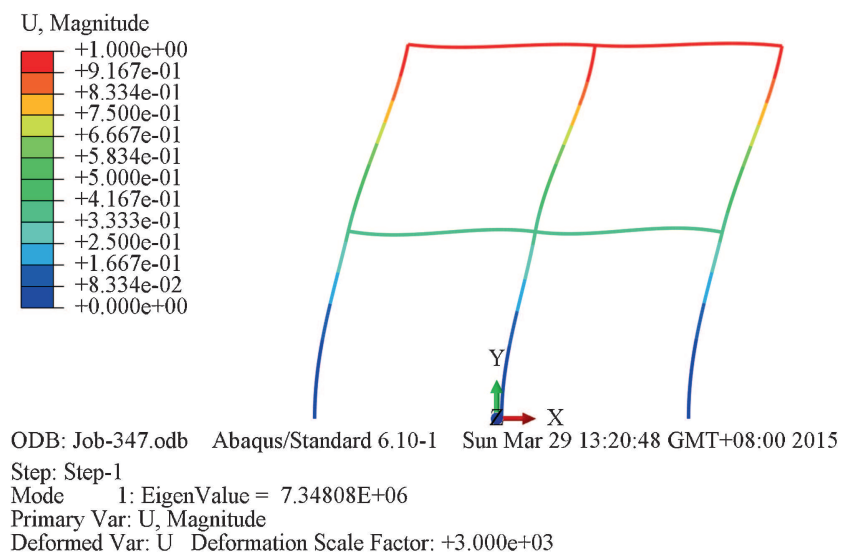

Figure 10. The buckling displacement of the median probabilistic result obtained by ABAQUS.

complex structure can be carried out by combining the professional FEM software with the proposed method.

\section{Conclusions}

This paper presented an evidence theory and differential evolution-based method to quantify and propagate mixed epistemic-aleatory uncertainty in uncertainty analyses. Evidence theory 
can describe many types of uncertainty in practical engineering more flexibly. As a novel evolutionary computation technique, differential evolution is used to solve two interval optimization problems in the process of uncertainty propagation of evidence theory, which can greatly increase calculation efficiency. The buckling load of a semi-rigid jointed frames problem provided two realistic examples of the epistemic treatment of the design uncertainties (initial connections' rigidity, bracing rigidity, elastic modulus, and applied load) to show that the proposed method is a promising approach for analyzing and propagating uncertainty in a structural system, whatever the types of uncertainty encountered.

\section{Acknowledgements}

This study was supported by the Ministry of Science and Technology of China, Grant No. SLDRCE14-B-03, SLDRCE11-B-01 and the National Natural Science Foundation of China, Grant No. 50708076.

\section{References}

Alibrandi U, Impollonia N and Ricciardi G 2009 Probabilistic Buckling Analysis of frame structures with uncertain parameters, XIX Congresso Aimeta, Ancona

Azizinamini A, Bradburn J H and Radziminski J B 1987 Initial stiffness of semi-rigid steel beam-to-column connections. J. Constr. Steel Res. 8: 71-90

Bae H R and Ramana V G 2004 Epistemic uncertainty quantification techniques including evidence theory for large-scale structures. Comput. Struct. 82: 1101-1112

Basaga H B, Kartal M E and Bayraktar A 2012 Reliablity analysis of steel braced reinforced concrete frames with semi-rigid connections. Int. J. Struct. Stab. Dyn 12(4): 1250037-1-1250037-20

Ben-Haim Y 2006 Info-Gap Theory: Decisions under severe uncertainty, Second edition. London: Academic Press

Byeng D Y, Choi K, Liu D and David G 2007 Integration of possibility-based optimization to robust design for epistemic uncertainty. ASME J. Mech. Des. 129: 876-883

Das S and Suganthan P N 2011 Differential evolution: a survey of the state-of-the-art. IEEE Trans. Evol. Comput. 15(1): 4-31

Dempster A P 1967 Upper and lower probabilities induced by multi-valued mapps. Annals of Math. Star. 38: $325-329$

Du L, Choi K K, Youn B D and Gorsich D 2006 Possibility-based design optimization method for design problems with both statistical and fuzzy input data. ASME J. Mech. Des. 128: 928-935

Dubois D and Prade H 1996 Possibility theory. New York: Plenum press

Eurocode 32005 Design of steel structures, Part 1-8: design of joints. BS EN 1993-1-8

Gao L and Haldar A 1995 Safety evaluation of frames with PR connections. ASCE J. Struct. Eng. 7(121): 1101-1109

Goto Y and Miyashita S 1998 Classification system for rigid and semirigid connections. ASCE J. Struct. Eng. 124(5): 750-757

Hadianfard M A and Razani R 2003 Effects of semi-rigid behavior of connections in the reliability of steel frames. Struct. Safe. 25: 123-138

Hanss M and Turrin S 2010 A fuzzy-based approach to comprehensive modeling and analysis of systems with epistemic uncertainties. Struct. Safe 32: 433-441

Helton J C, Johnson J D, Oberkampf W L and Sallaberry C J 2010 Representation of analysis results involving aleatory and epistemic uncertainty. Int. J. Gen. Syst. 39(6): 605-646

Helton J C and Oberkampf W L 2004 Alternative representations of epistemic uncertainty. Reliab. Eng. Syst. Safe. 85: 1-10 
Huh J and Haldar A 2001 Seismic risk analysis of frames with uncertain support and PR connection conditions. Struct. Eng. 4(4): 329-338

Jiang C, Wang B, Li Z R, Han X and Yu D J 2013 An evidence-theory model considering dependence among parameters and its application in structural reliability analysis. Eng. Struct 57: 12-22

Korkmaz K, Demir F and Tekeli H 2011 Uncertainty modeling of critical column buckling for reinforced concrete buildings. Sadhana 36(2): 267-280

Li M and Azarm S 2008 Multiobjective collaborative robust optimization with interval uncertainty and interdisciplinary uncertainty propagation. ASME J. Mech. Des 130: 81402-81412

Lien K H, Chiou Y J and Hsiao P A 2012 Vector form intrinsic finite-element analysis of analysis of steel frames with semirigid joints. ASCE J. Struct. Eng. 138(3): 327-336

Limbourg P and Rocquigny E D 2010 Uncertainty analysis using evidence theory-confronting level-1 and level-2 approaches with data availability and computational constraints. Reliab. Eng. Syst. Safe. 95(4): $550-564$

Modares M, Mullen R, Muhanna R L and Zhang H 2004 Buckling analysis of structures with uncertain properties and loads using an interval finite element method. Proceedings of the NSF Workshop on Reliable Engineering. Savannah, GA: Georgia Tech

Moncarz P D and Gerstle K H 1981 Steel Frames with non-linear connections. J. struct. Div. 107, ST8: $1427-1441$

Muhanna R L and Mullen R L 2001 Uncertainty in mechanics problems?interval-based approach. ASME J. Eng. Mech. 127: 557-566

Oberkampf W L and Helton J C 2001 Mathematical representation of uncertainty. Non-deterministic approaches Forum, Seattle, WA; AIAA-20011645

Raftoyiannis I G 2005 The effect of semi-rigid joints and an elastic bracing system on the buckling load of simple rectangular steel frames. J. Constr. Steel Res. 61: 1205-1225

Rauscher T R and Gerstle K H 1992 Reliability of rotational behavior of framing connections. Eng. J. AISC 29(1): 12-9

Sakurai S and Ellingwood R B 2001 Probabilistic study of the behavior of steel frames with partially restrained connections. Eng. Struct. 23: 1410-1417

Savage L J 1972 The foundations of statistics. New York: Dover Publications

Sentz K and Ferson S 2002 Combination of evidence in Dempster-Shafer theory. Sandia National. Laboratories SAND 2002-0835

Shafer G 1976 A mathematical theory of evidence. New Jersey: Princeton University Press

Storn R and Price K 1997 Differential evolution-A simple and efficient adaptive scheme for global optimization over continuous spaces. J. Glob. Optim. 11: 341-359

Tang H, Xue S and Fan C 2008 Differential evolution strategy for structural system identification. Comput. Struct. 86(21-22): 2004-2012

Tucker G L and Bennett R M 1990 Reliability analysis of partially restrained steel connections. ASCE J. Struct. Eng. 116: 1090-1101

Walpole R E 1998 Probability and statistics for engineers and scientists. New Jersey: Prentice Hall

Yee Y and Melchers R 1987 Moment-rotation curves for bolted connections. ASCE J. Struct. Eng. 112(3): $615-635$ 